\title{
Synthesis and characterization of novel 1,8-Naphthalimide derivatives containing 1,3-oxazoles, 1,3-thiazoles, 1,2,4-triazoles as antimicrobial agents
}

\author{
Suaad M. H. Al-Majidi*, Mohammed R. Ahmad* and Ayad Kareem Khan ${ }^{* *}$ \\ *Department of Chemistry, College of Science, Baghdad University, Baghdad-Iraq. \\ ** Department of Pharmaceutical Chemistry, College of Pharmacy, Al-Mustansiriyah University, \\ Baghdad-Iraq. \\ E-mail: kareem_ayad@yahoo.com.
}

\begin{abstract}
This research include developing new heterocyclic derivatives of 1,8-naphthalimides bearing 1,3-oxazole, 1,3-thiazole and 1,3,4-triazole moieties as the following:

Direct imidation of 1,8-naphthalic anhydride with ethylglycinate in dimethylsulfoxide as solvent under reflux at high temperature for sixteen hours to obtain the $N$-ester-1,8-naphthalimide(1). Then conversion of this ester into (urea, thiourea, semicarbazide, phenylsemicarbazide, thiosemicarbazide and phenylthiosemicarbazide) derivatives through its reaction with (urea, thiourea, semicarbazide, phenylsemicarbazide, thiosemicarbazide and phenylthiosemicarbazide) respectively to give compounds $(2,6,10,12,14$ and16). Then cyclization of these compounds by using different reagents. The first cyclization of compounds ( 2 and 6$)$ by using $p$-substituted phenacylbromide to give oxazole derivatives (3-5) and thiazole derivatives (7-9) respectively. Furthermore triazole derivatives were prepared through the second cyclization of compounds (10,12,14 and16) in alkaline media(4N. NaOH) to give compounds $(11,13,15$ and 17$)$ respectively.

The structure of the newly synthesized compounds was identified by their FTIR, and some of them by ${ }^{1} \mathrm{H}-\mathrm{NMR},{ }^{13} \mathrm{C}-\mathrm{NMR}$ spectral data and some physical properties and some specific reactions.

Also new compounds were screened in three concentration for their in vitro antimicrobial activity against both Gram (+ve) such as Staphylococcus aureus, Bacillus and Gram (-ve) Escherichia Coli, pseudomonas aeuroginosa bacteria and against Candida albicans fungal and they were found to exhibit good to moderate antimicrobial activities.
\end{abstract}

Keywords: 1,8-naphthalimides, 1,3-oxazole, 1,3-thiazole and 1,2,4 triazole, synthesis , antimicrobial activity.

\section{Introduction}

Naphthalimides, one type of cyclic imides [1] with strong hydrophobicity and desirable large $\pi$-conjugated backbone, could easily interact with various active targets in biological system via non-covalent forces such as $\pi-\pi$ stacking, and exhibit diverse biological activities including anticancer [2], antibacterial [3], antitrypanosomal[4], analgesic potency [5]. 1,8-Naphthalimides are well-known as broad-spectrum activity against a variety of human solid tumor cells [6]. Several derivatives have reached the phases of clinical trials [7].

The azole moiety is an important structural feature of many biologically active compounds [8]. Various 1,3-oxazole functional group associated biological activities [9],[10] . More thiazole ring system is an important class of compounds in medicinal chemistry [11]. This structure has found applications in drug development. A number of thiazole derivatives have been reported to possess significant and diverse biological activities [12],[13]. Moreover, 1,2,4-triazole and their derivatives have been found to be associated with diverse agricultural, industrial and pharmacological activities [14], [15].

In this connection, the synthesis of 1,8 naphthalimide derivatives containing five membered ring substituent, in particular 1,3oxazole, 1,3-thiadiazole, and 1,2,4-triazole fragments which could considerably affect biological properties of 1,8-naphthalimide, to develop novel and potent therapeutic agents of synthetic origin, it was decided to synthesize certain these derivatives and evaluate them for their antimicrobial properties 


\section{Experimental}

Materials and Instruments

Chemicals used in this work are supplied from Merck, Sigma-Aldrich, BDH and Fluka companies and are used without further purification.

Melting points were recorded using digital Stuart Scientific SMP3 melting point apparatus and are uncorrected. FTIR spectra were recorded on SHIMADZU FTIR-8400 Fourier Transform Infrared spectrophotometer using $\mathrm{KBr}$ discs in the (500-4000) $\mathrm{cm}^{-1}$ spectral range. ${ }^{1} \mathrm{HNMR}$ and ${ }^{13} \mathrm{CNMR}$ spectra were recorded on Bruker $300 \mathrm{MHz}$ instrument using DMSO- $\mathrm{d}^{6}$ as a solvent and TMS as internal reference. Thin layer chromatography (TLC) was carried out using Fertigfollen precoated sheets type Polygram Silg, and the plates were developed with iodine vapour. The antimicrobial activity was performed in clinical laboratory department, college of pharmacy, Al-Mustansiriyah University.

\section{Synthesis of $N$-Ethylglycinate-1,8-} naphthalimide(1).

$(0.005 \mathrm{~mol}, \quad 1 \mathrm{~g})$ of 1,8-Naphthalic anhydride was dissolved in $30 \mathrm{~mL}$ dimethyl sulfoxide with stirring and heating. (0.006 mol, $0.837 \mathrm{~g}$ ) ethyl glycinate hydrochloride after neutralized with dilute solution of sodium bicarbonate was added and the mixture was refluxed until TLC showed no 1,8-naphthalic anhydride remained. This reaction was completed in (16 hrs). The mixture was then poured into ice water. The yellow precipitated solid was filtered off and recrystallized from ethanol [16].

Synthesis of (1,8-naphthalimide $N$-yl)acetourea(2), thiourea(6), semicarbazide(10), phenylsemicarbazide(12)

thiosemicarbazide(14), and

phenylthiosemicarbazide (16) respectively:

A mixture of ester (1) $(0.0035 \mathrm{~mol}, 1 \mathrm{~g})$ with (urea, thiourea, semicarbazide, phenylsemicarbazide, thiosemicarbazide, and phenylthiosemicarbazide) respectively $(0.0035$ mol) and sodium acetate $(0.0035 \mathrm{~mol}, 0.31 \mathrm{~g})$ in absolute ethanol $(30 \mathrm{ml})$ was refluxed for (10-16 hr). The reaction mixture was filtered and poured on ice water; the precipitate was filtered and recrystallized from suitable solvents to give crystals [17].
Synthesis of (1,8-naphthalimide $N$-yl) acetamido [4-( $p$-substituted phenyl)-1,3oxazole-2-yl)] (3-5) and (1,8-naphthalimide $N$-yl) acetamido [4-( $p$-substituted phenyl)1,3-thiazole-2-yl)] (7-9) .

A mixture of compound (2 or 6) $(0.0033 \mathrm{~mol})$ with absolute ethanol $(20 \mathrm{ml})$, $p$-substituted phenacyl bromide $(0.0033 \mathrm{~mol})$ was refluxed for (12-14 hrs.), cooled and neutralized with ammonium hydroxide solution. The precipitate was filtered off, washed with water, and suitable solvents were used for recrystallization [18].

Synthesis of $[(1,8$-naphthalimide $N$-yl) methyl]-1,2,4-triazol-3-ol (11), 1,2,4-triazol1-phenyl-3-ol (13), 1,2,4-triazol-3-thiol (15) and 1,2,4-triazol-1-phenyl-3-thiol (17).

In round bottom flask $(0.0032 \mathrm{~mol})$ for compounds [10,12,14 and 16] was refluxed with $20 \%$ aqueous sodium hydroxide solution (25ml) for (10-12 hrs.), cooled, poured on to ice water, stirred and neutralized by gradual addition of (1:1) hydrochloric acid. The formed precipitate was filtered and recrystallized from suitable solvents [19].

\section{Antimicrobial Activity test}

The test compounds were prepared with different concentrations (100, 50, and 25) $\mathrm{mg} / \mathrm{ml}$ using dimethyl sulfoxide (DMSO) as solvent. The agar well diffusion method was used to determine antimicrobial activity [20]. The culture medium was inoculated with one of tested bacteria or fungi suspended in nutrient broth. Six millimeter diameter wells punched into the agar with fresh bacteria or fungi separately and filled with $100 \mu$ l of each concentration. DMSO was used as control. The incubation was carried out at $37^{\circ} \mathrm{C}$ for $4 \mathrm{hr}$. Sulfamethxazole was used as a standard drug. Solvent and growth controls were kept and zones of inhibition were noted. The antibacterial activity was evaluated by measuring the inhibition zone diameter observed are recorded.

\section{Result and Discussion}

The synthetic sequences for preparation of series new 1,8-naphthalimides, 1,3-oxazole, 1,3-thiazole, and 1,2,4-triazole is outlined in Scheme (1). Naphthalic anhydride reacts with amines such as liquid ammonia or alkyl amines to form the corresponding 
naphthalimides. Therefore, 1,8- naphthalic anhydride have been used as conventional starting material for preparation of 1,8naphthalimides. Compound (1) were synthesized by condensation of the 1,8naphthalic anhydride was reacted with ethyl glycinate was carried out in dimethyl sulfoxide media under reflux condition, and the end point of the reaction was examined by thin layer chromatography(TLC). TLC showed the imidation of 1,8-naphthalic anhydride with ethyl glycinate completed at 16 hours. The time required for completion of the imidation reaction for 1,8-naphthalic anhydride with ethyl glycinate is more than for the imidation of 1,8-naphthalic anhydride with alkyl amines [21],[22].

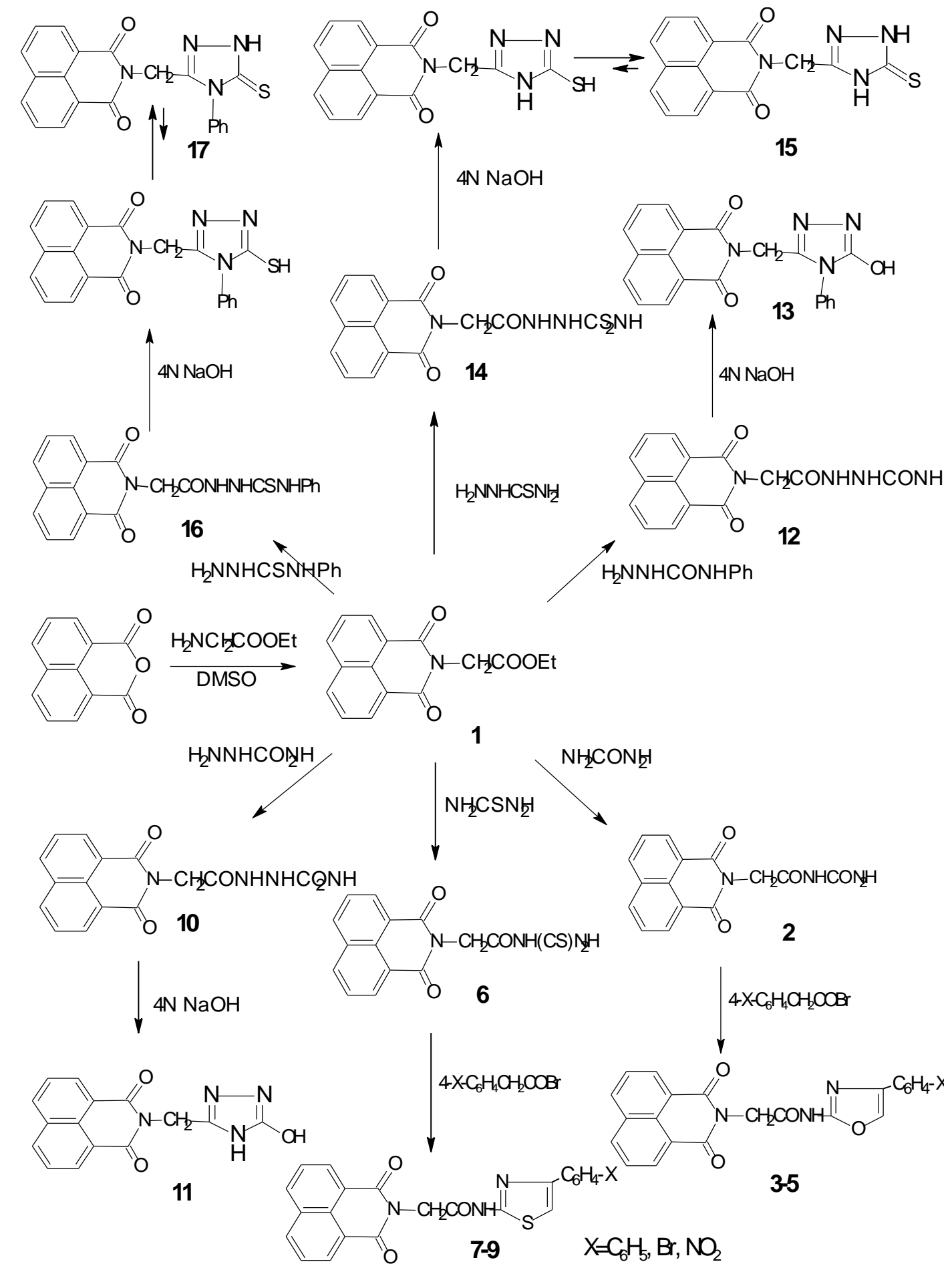

Scheme (1). 
This can be attributed to the alkyl amines being more active than the ethyl glycinates in the nucleophilic displacement reaction in which the attacking group is amine. Imidation process of 1,8-naphthalic anhydride with ethyl glycinate as show in the Scheme (1).

Compound (1) was afforded in good yield (76\%), having melting point $(250-252){ }^{\circ} \mathrm{C}$ Hydroxamic acid test give (+ve) for presence of ester [23]. Physical properties of compound (1) are listed in Table (1). FTIR spectrum showed clear absorption bands at (1774) $\mathrm{cm}^{-1}$, due to $v(\mathrm{C}=\mathrm{O})$ ester, $(1701,1668) \mathrm{cm}^{-1}$ due to $v(\mathrm{C}=\mathrm{O})$ imide. Other absorption bands appeared at (1581) $\mathrm{cm}^{-1}$, (1357) $\mathrm{cm}^{-1}$, and (1211) $\mathrm{cm}^{-1}$ due to $v(\mathrm{C}=\mathrm{C})$ aromatic, $v(\mathrm{C}-\mathrm{N})$ imide and $v(\mathrm{C}-\mathrm{O}-\mathrm{C})$ ester respectively. ${ }^{1} \mathrm{HNMR}$ spectrum of the same compound (1) showed triplet signal at $\delta=(1.19-1.27) \mathrm{ppm}$ due to $\left(\mathrm{CH}_{3}\right)$ protons, singlet signal at $\delta=$ (4.08) ppm belong to $\left(\mathrm{N}-\mathrm{CH}_{2}-\mathrm{CO}-\right)$ protons, quartate signal at $\delta=(4.50-4.58) \mathrm{ppm}$ due to $\left(-\mathrm{O}-\mathrm{CH}_{2}-\right)$ protons, and signals at $\delta=(7.04-$ 7.75) ppm due to aromatic protons, Fig.(1). ${ }^{13} \mathrm{CNMR}$ spectrum of this compound (1) showed results were listed in Table (4), Fig.(2).

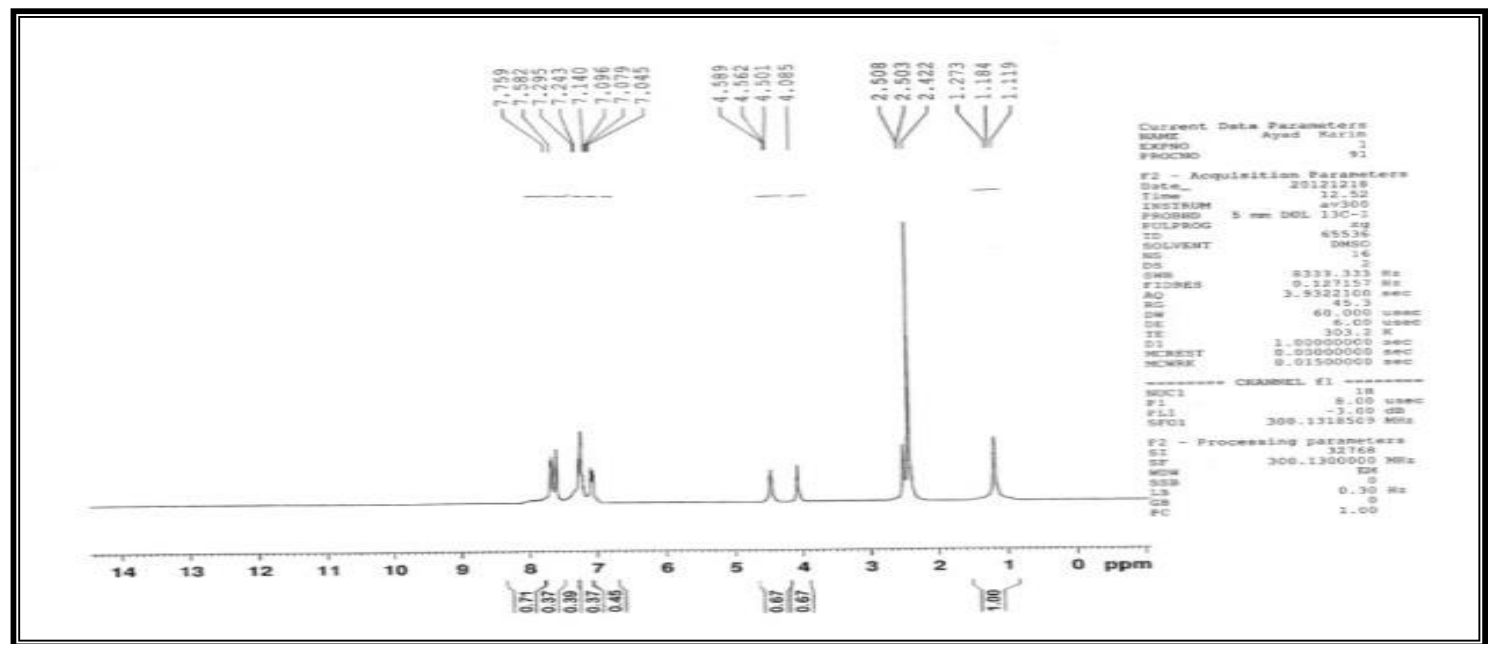

Fig. (1) ${ }^{1}$ HNMR Spectra for compound (1).

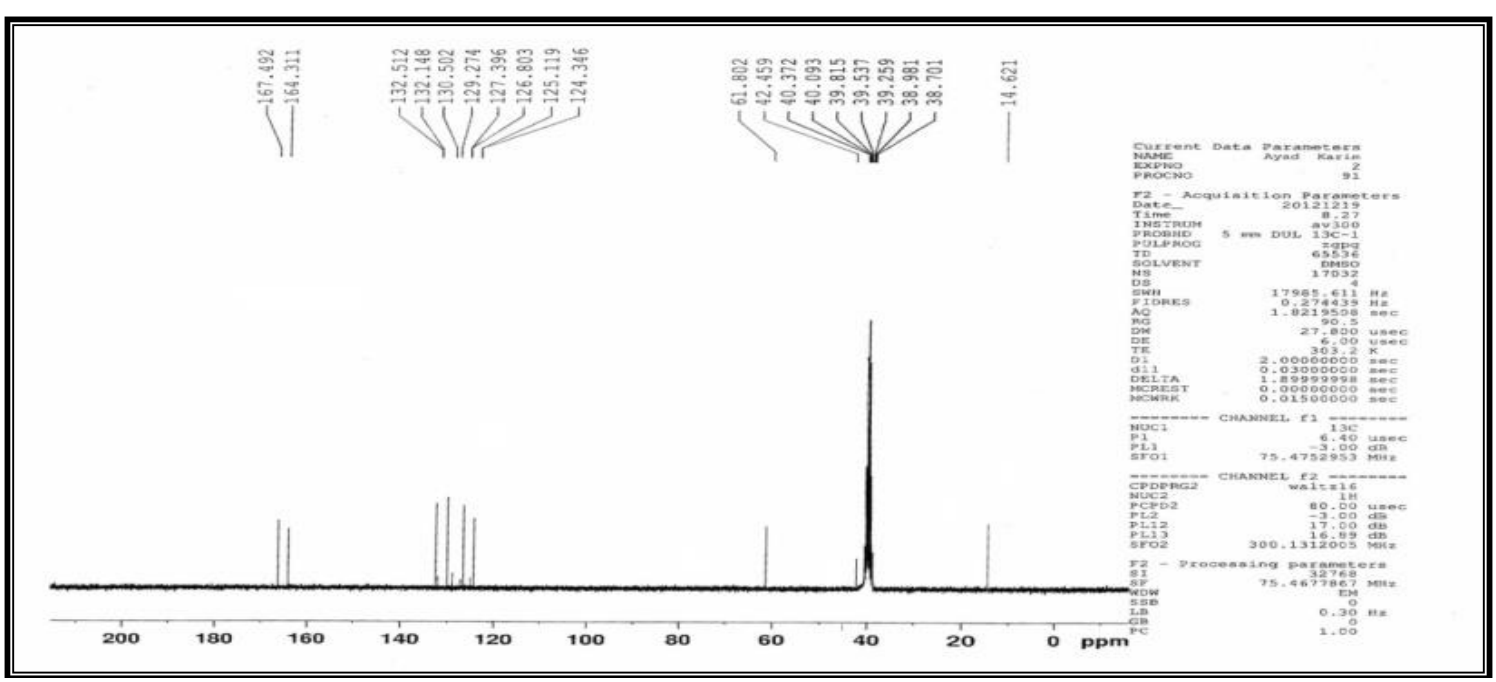

Fig. (2) ${ }^{13}$ CNMR Spectra for compound (1).

The ester (1) was converted to \{urea (2), thiourea (6), semicarbazide (10), phenylsemicarbazide (12), thiosemicarbazide (14) and phenylthiosemicarbazide (16)\} derivatives by reaction with (urea, thiourea, semicarbazide, thiosemicarbazide phenylsemicarbazide, and phenylthio- semicarbazide) respectively in absolute ethanol Scheme (1). FTIR spectral date showing the absorption at $3544 \mathrm{~cm}^{-1}$ asym. $3498 \mathrm{~cm}^{-1}$ sym. for $\mathrm{NH}_{2}, 1747 \mathrm{~cm}^{-1}$ for $\mathrm{C}=\mathrm{O}$ amide of compound (2). $3508 \mathrm{~cm}^{-1}$ asym. $3430 \mathrm{~cm}^{-1}$ sym. for $\mathrm{NH}_{2}, 1747 \mathrm{~cm}^{-1}$ for $\mathrm{C}=\mathrm{O}$ amide $1240 \mathrm{~cm}^{-1}$ for $\mathrm{C}=\mathrm{S}$, of compound (6). 
$3425 \mathrm{~cm}^{-1}$ asym. $3309 \mathrm{~cm}^{-1}$ sym. for $\mathrm{NH}_{2}$ $1748 \mathrm{~cm}^{-1}$ for $\mathrm{C}=\mathrm{O}$ amide of compound (10). $3338 \mathrm{~cm}^{-1}$ for $\mathrm{NH}, 1747 \mathrm{~cm}^{-1}$ for $\mathrm{C}=\mathrm{O}$ amide of compound (12). $3416 \mathrm{~cm}^{-1}$ asym. $3367 \mathrm{~cm}^{-1}$ sym. for $\mathrm{NH}_{2}, 1747 \mathrm{~cm}^{-1}$ for $\mathrm{C}=\mathrm{O}$ amide, $1284 \mathrm{~cm}^{-1}$ for $\mathrm{C}=\mathrm{S}$, of compound (14). 3244 $3338 \mathrm{~cm}^{-1}$ for $\mathrm{NH}, 1748 \mathrm{~cm}^{-1}$ for $\mathrm{C}=\mathrm{O}$ amide, $1243 \mathrm{~cm}^{-1}$ for $\mathrm{C}=\mathrm{S}$, of compound (16). Physical properties of these compounds are listed in Table (1) and Table (2).

Treatment of compound (2) and (6) with $p$-substituted phenacylbromide afford intramolecular cyclization to give the oxazoles (3-5) and thiazoles (7-9).

The FTIR spectrum of compounds (3-5) showed absorption bands between (3464$3427) \mathrm{cm}^{-1}$ for $\mathrm{NH},(1747-1748) \mathrm{cm}^{-1}$ for $\mathrm{C}=\mathrm{O}$ amide, (1705-1666) $\mathrm{cm}^{-1}$ for $\mathrm{C}=\mathrm{O}$ imide, (1600-1608) $\mathrm{cm}^{-1}$ for $\mathrm{C}=\mathrm{N}$, and others $605 \mathrm{~cm}^{-1}$ for C-Br (4), (1535 asym. $1431 \mathrm{sym}$.) $\mathrm{cm}^{-1}$ for $\mathrm{NO}_{2}$ (5) and disappearance the absorption band of $\left(\mathrm{NH}_{2}\right)$ group.
${ }^{1}$ HNMR spectrum of compound [3] showed signal at $\delta=4.27 \mathrm{ppm}$ belong to $\left(\mathrm{N}-\underline{\mathrm{CH}}_{2}-\mathrm{CO}-\right)$ protons, $\delta=5.84 \mathrm{ppm}\left(\mathrm{C}_{5}\right)$ of oxazole ring proton, $\delta=(6.85-7.72) \mathrm{ppm}$ aromatic ring protons, $\delta=8.32 \mathrm{ppm}(\mathrm{NH})$ proton. Figure (3). ${ }^{13} \mathrm{CNMR}$ spectrum of this compound (3) showed results were listed in Table (4), Fig.(4).

FTIR spectrum of compound (7-9) showed absorption bands between (3348-3360) $\mathrm{cm}^{-1}$ for $\mathrm{NH},(1746-1735) \mathrm{cm}^{-1}$ for $\mathrm{C}=\mathrm{O}$ amide, (1700-1638) $\mathrm{cm}^{-1}$ for $\mathrm{C}=\mathrm{O}$ imide, (16121601) $\mathrm{cm}^{-1}$ for $\mathrm{C}=\mathrm{N}$, and others $613 \mathrm{~cm}^{-1}$ for C-Br (8), (1558 asym.1473 sym.) $\mathrm{cm}^{-1}$ for $\mathrm{NO}_{2}(9)$ and disappearance the absorption $\left(\mathrm{NH}_{2}\right.$ and $\mathrm{C}=\mathrm{S}$ ) groups.

${ }^{1} \mathrm{HNMR}$ spectrum of compound (8) showed results were listed in Table (3) and ${ }^{13} \mathrm{CNMR}$ spectrum of compound (8) showed results were listed in Table (4).

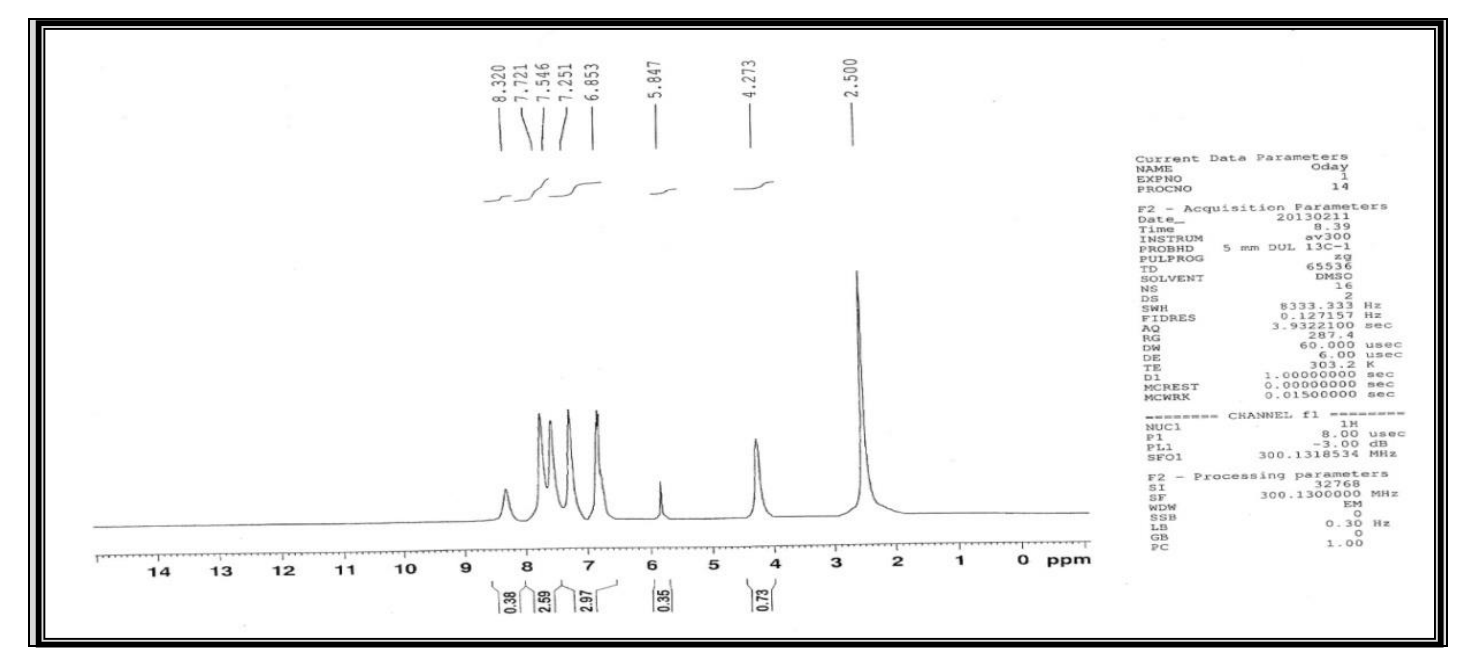

Fig. (3) ${ }^{1}$ HNMR Spectra for compound (3).

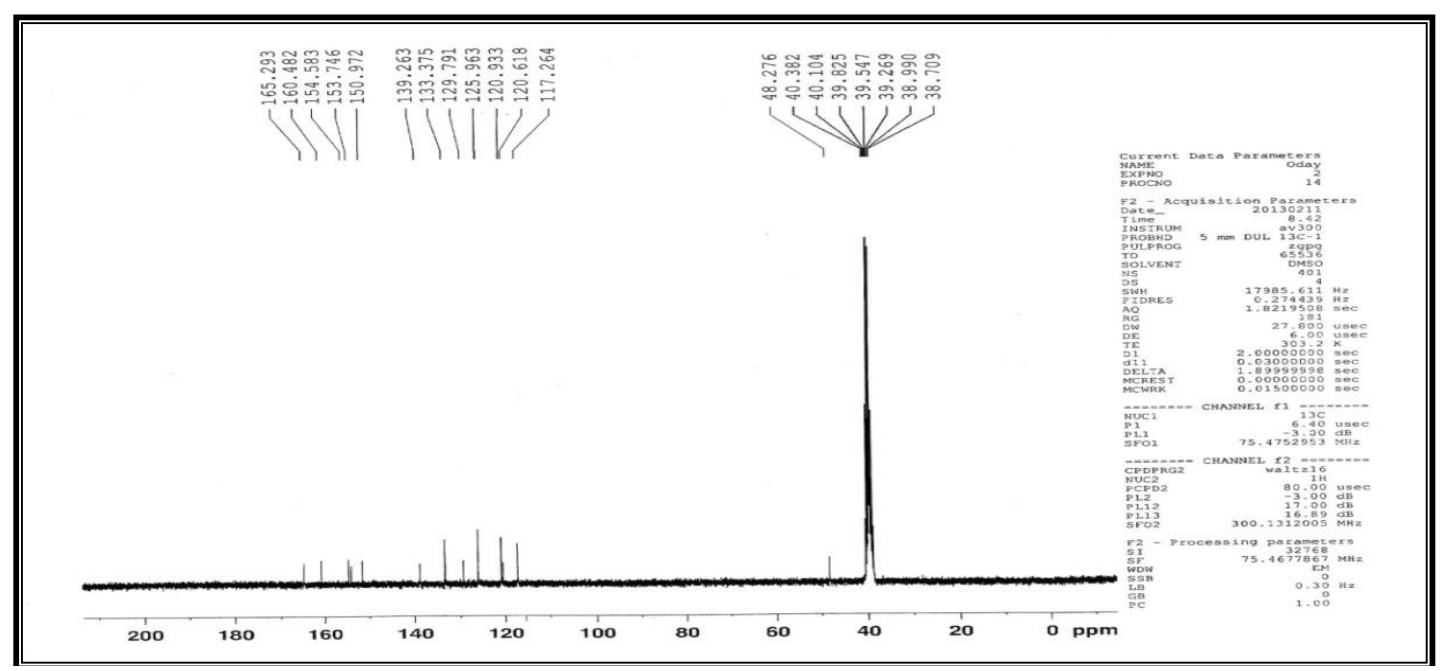

Fig. (4) ${ }^{13}$ CNMR Spectra for compound (3). 
Treatment of compounds $(10,12,14$ and 16) with (4N.NaOH) solution afford intramolecular cyclization to give the hydroxytriazole (11), Phenylhydroxy-triazole (13), thiotriazole (15) and thiohydroxytriazole
(17) were identified from FTIR spectra shows results listed in Table (2). ${ }^{1} \mathrm{HNMR}$ and ${ }^{13} \mathrm{CNMR}$ spectrum of these compounds showed results were listed in Table (3) and (4) respectively.

Table (1)

Physical properties and FTIR spectral data $\mathrm{cm}^{-1}$ of compounds (1-9).

\begin{tabular}{|c|c|c|c|c|c|c|c|c|c|}
\hline \multicolumn{5}{|c|}{ Physical properties } & \multicolumn{5}{|c|}{ Major FTIR Absorption $\mathrm{cm}^{-1}$} \\
\hline $\begin{array}{c}\text { Comp. } \\
\text { No. }\end{array}$ & Compound structure & Color & $\begin{array}{c}\text { Yield } \\
\%\end{array}$ & $\begin{array}{c}\begin{array}{c}\text { Melting } \\
\text { Point } \\
{ }^{\circ} \mathrm{C}\end{array} \\
\end{array}$ & $\begin{array}{c}v \\
(N H)\end{array}$ & $\begin{array}{c}v \\
(C=O) \\
\text { amide }\end{array}$ & $\begin{array}{c}v \\
(C=O) \\
\text { imide }\end{array}$ & $\begin{array}{c}v \\
(C-N) \\
\text { imide }\end{array}$ & Others \\
\hline 1 & & $\begin{array}{l}\text { Yellow- } \\
\text { green }\end{array}$ & 76 & $250-252$ & - & - & $\begin{array}{l}1701 \\
1668\end{array}$ & 1357 & $\begin{array}{c}v(C=O) \\
\text { ester 1774, } \\
v(C-O-C) \\
\text { ester 1211 }\end{array}$ \\
\hline 2 & & $\begin{array}{c}\text { Off } \\
\text { white }\end{array}$ & 80 & 111-114 & 3414 & 1747 & $\begin{array}{l}1701 \\
1666\end{array}$ & 1384 & $\begin{array}{c}v\left(\mathrm{NH}_{2}\right) \\
\text { Asym.3544, } \\
\text { sym.3498 }\end{array}$ \\
\hline 3 & & Dusty & 71 & $164-166$ & 3464 & 1747 & $\begin{array}{l}1705 \\
1685\end{array}$ & 1384 & $\begin{array}{c}v(C=N) \\
1600\end{array}$ \\
\hline 4 & & Violet & 60 & $135-137$ & 3427 & 1747 & $\begin{array}{l}1705 \\
1666\end{array}$ & 1384 & $\begin{array}{c}v(\mathrm{C}=\mathrm{N}) \\
1608, \\
v(\mathrm{C}-\mathrm{Br}) \\
\quad 605\end{array}$ \\
\hline 5 & & $\begin{array}{l}\text { Pale- } \\
\text { yellow }\end{array}$ & 74 & $153-155$ & 3458 & 1748 & $\begin{array}{l}1705 \\
1666\end{array}$ & 1392 & $\begin{array}{c}v(\mathrm{C}=\mathrm{N}) \\
1608, \\
v\left(\mathrm{NO}_{2}\right) \\
1535,1431\end{array}$ \\
\hline 6 & & $\begin{array}{c}\text { Light } \\
\text { brown }\end{array}$ & 69 & 194-196 & 3380 & 1747 & $\begin{array}{l}1705 \\
1666\end{array}$ & 1384 & $\begin{array}{c}v\left(\mathrm{NH}_{2}\right) \\
\text { Asym.3508, } \\
\text { sym.3430 } \\
v(\mathrm{C}=\mathbf{S}) \\
\mathbf{1 2 4 0}\end{array}$ \\
\hline 7 & & Brown & 77 & 202-205 & 3348 & 1735 & $\begin{array}{l}1700 \\
1658\end{array}$ & 1373 & $\begin{array}{c}v(\mathrm{C}=\mathrm{N}) \\
1612\end{array}$ \\
\hline 8 & & Brown & 84 & 187-189 & 3258 & 1746 & $\begin{array}{l}1701 \\
1668\end{array}$ & 1354 & $\begin{array}{c}v(\mathrm{C}=\mathrm{N}) \\
1601, \\
v(\mathrm{C}-\mathrm{Br}) \\
613\end{array}$ \\
\hline 9 & & Yellow & 81 & 179-181 & 3360 & 1742 & $\begin{array}{l}1700 \\
1638\end{array}$ & 1396 & $\begin{array}{c}v(\mathrm{C}=\mathrm{N}) \\
1600 \\
v\left(\mathrm{NO}_{2}\right) \\
1558,1473\end{array}$ \\
\hline
\end{tabular}


Table (2)

Physical properties and FTIR spectral data $\mathrm{cm}^{-1}$ of compounds (10-17).

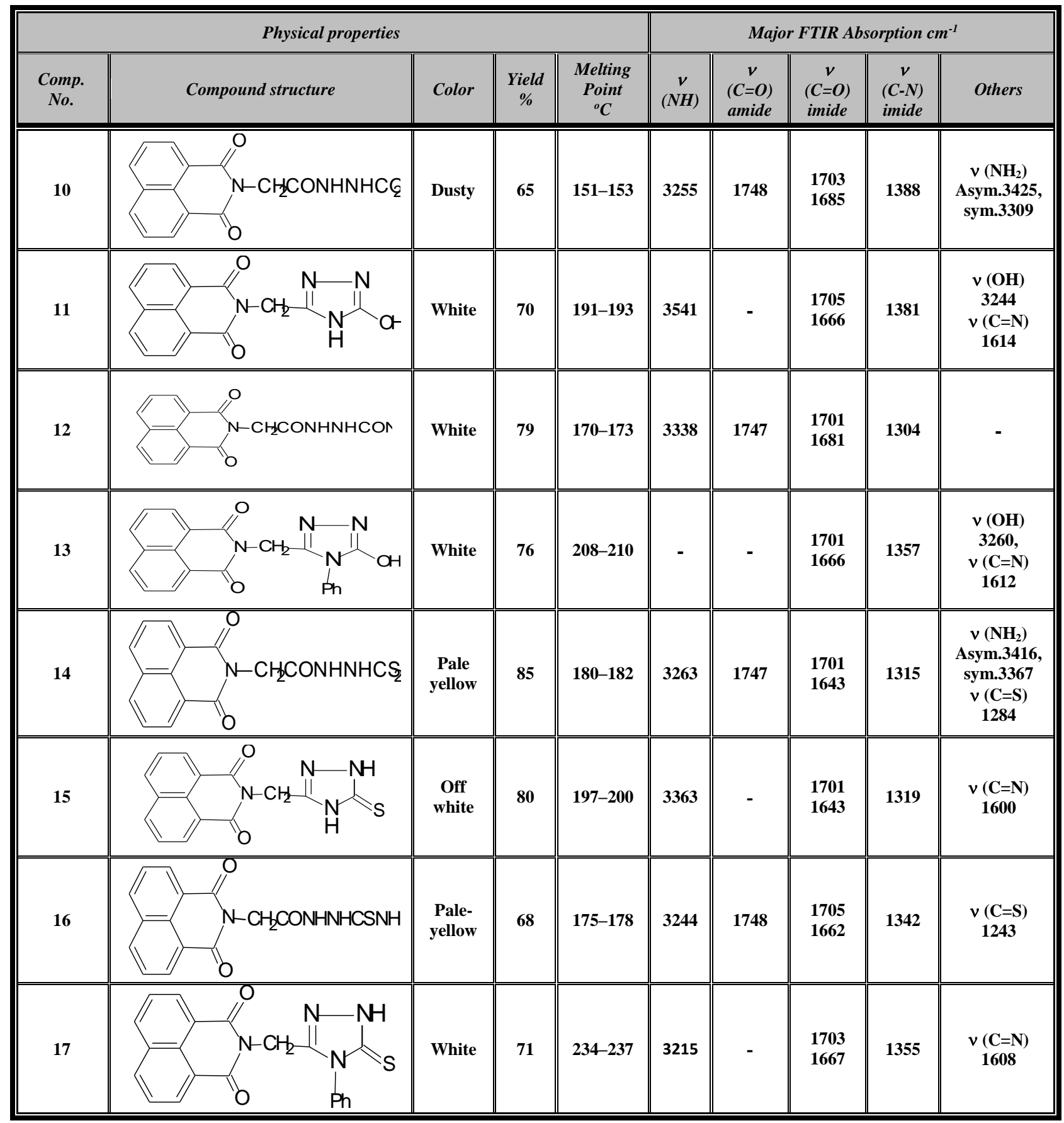


Table (3)

${ }^{1}$ HNMR spectral data $(\delta$ ppm) for selected compounds.

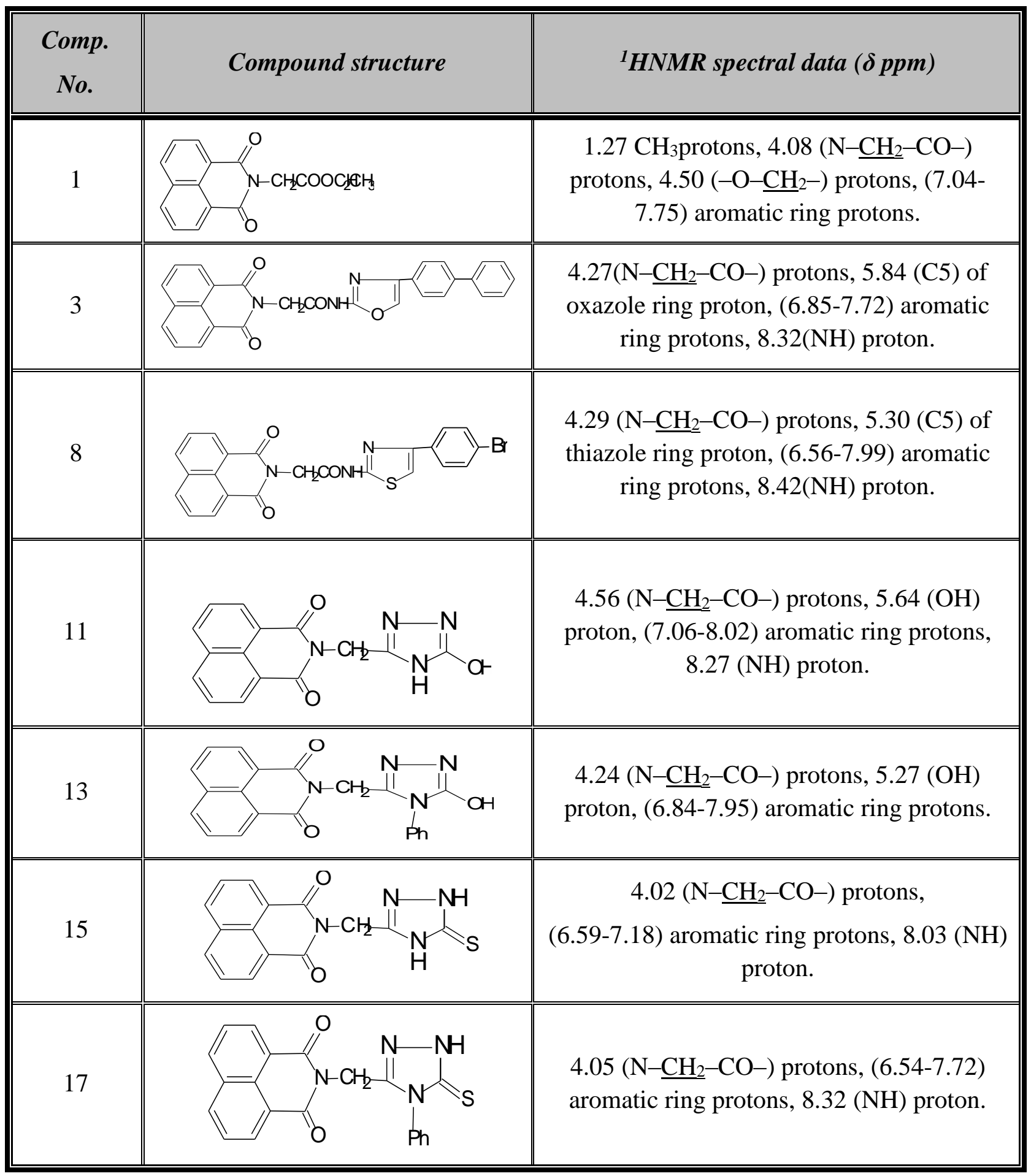


Table (4)

${ }^{13}$ CNMR spectral data ( $\delta$ ppm) for selected compounds.

\begin{tabular}{|c|c|c|}
\hline $\begin{array}{c}\text { Comp. } \\
\text { No. }\end{array}$ & Compound structure & ${ }^{13} \mathrm{CNMR}$ spectral data $(\delta \mathrm{ppm})$ \\
\hline 1 & 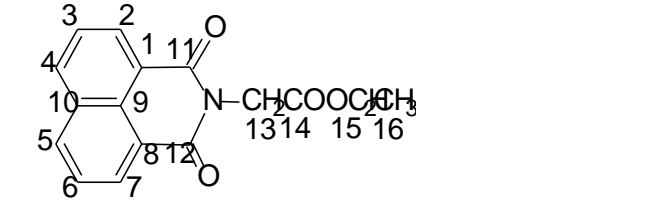 & $\begin{array}{c}14.62\left(\mathrm{C}_{16}\right), 42.45\left(\mathrm{C}_{15}\right), 61.6\left(\mathrm{C}_{13}\right), \\
124.34-132.51\left(\mathrm{C}_{1}-\mathrm{C}_{10}\right), 164.31\left(\mathrm{C}_{11}, \mathrm{C}_{12}\right), \\
167.49\left(\mathrm{C}_{14}\right) .\end{array}$ \\
\hline 3 & 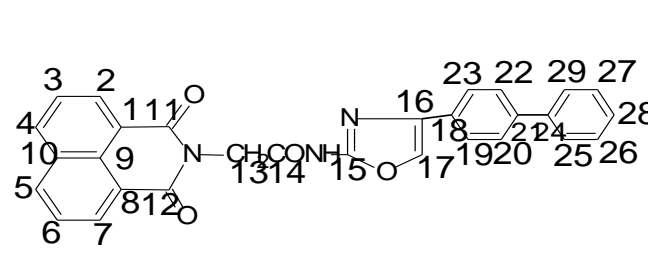 & $\begin{array}{c}48.27\left(\mathrm{C}_{13}\right) \\
\left.\text { 117.26-139.25( } \mathrm{C}_{1-}-\mathrm{C}_{10}\right) \text { and }\left(\mathrm{C}_{18}-\mathrm{C}_{29}\right) \\
\left.\text { 150.97( } \mathrm{C}_{16}\right), 153.74\left(\mathrm{C}_{17}\right), 154.58\left(\mathrm{C}_{15}\right) \\
160.48\left(\mathrm{C}_{11}, \mathrm{C}_{12}\right), 165.29\left(\mathrm{C}_{14}\right)\end{array}$ \\
\hline 8 & 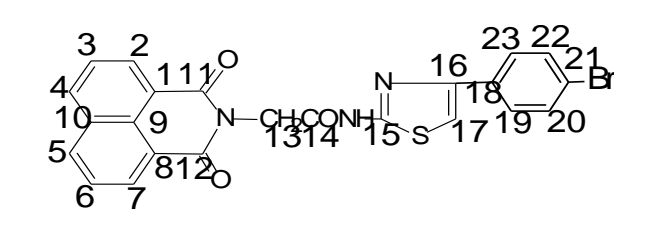 & $\begin{array}{c}49.80\left(\mathrm{C}_{13}\right), \\
117.53-137.11\left(\mathrm{C}_{1}-\mathrm{C}_{10}\right) \text { and }\left(\mathrm{C}_{18}-\mathrm{C}_{23}\right) \\
154.91\left(\mathrm{C}_{17}\right), 158.68\left(\mathrm{C}_{16}\right), 160.83\left(\mathrm{C}_{15}\right) \\
161.19\left(\mathrm{C}_{11}, \mathrm{C}_{12}\right), 166.52\left(\mathrm{C}_{14}\right)\end{array}$ \\
\hline 11 & 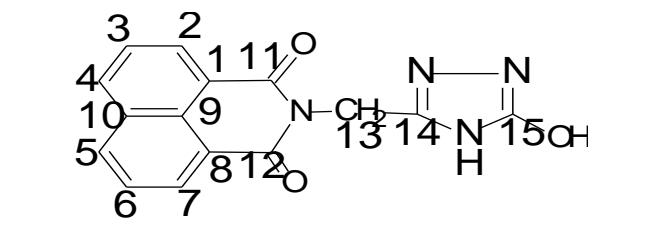 & $\begin{array}{c}46.71\left(\mathrm{C}_{13}\right), 125.68-131.47\left(\mathrm{C}_{1}-\mathrm{C}_{10}\right), \\
151.39\left(\mathrm{C}_{14}\right), 156.40\left(\mathrm{C}_{15}\right), \\
162.66\left(\mathrm{C}_{11}, \mathrm{C}_{12}\right)\end{array}$ \\
\hline 13 & 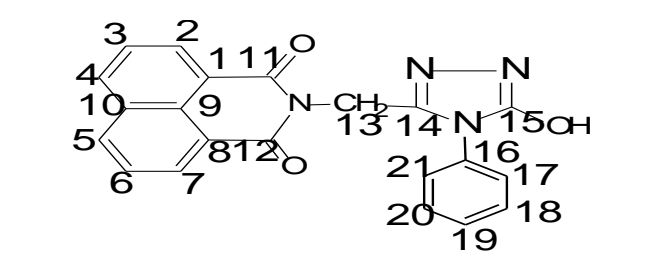 & $\begin{array}{c}44.10\left(\mathrm{C}_{13}\right), 119.63-133.91\left(\mathrm{C}_{1-} \mathrm{C}_{10}\right) \text { and } \\
\left(\mathrm{C}_{16}-\mathrm{C}_{21}\right), 154.38\left(\mathrm{C}_{14}\right), 158.02\left(\mathrm{C}_{15}\right) \\
163.52\left(\mathrm{C}_{11}, \mathrm{C}_{12}\right)\end{array}$ \\
\hline 15 & (812 & $\begin{array}{c}46.13\left(\mathrm{C}_{13}\right), 124.43-130.86\left(\mathrm{C}_{1}-\mathrm{C}_{10}\right) \\
159.91\left(\mathrm{C}_{14}\right), 161.35\left(\mathrm{C}_{11}, \mathrm{C}_{12}\right) \\
176.24\left(\mathrm{C}_{15}\right)\end{array}$ \\
\hline 17 & 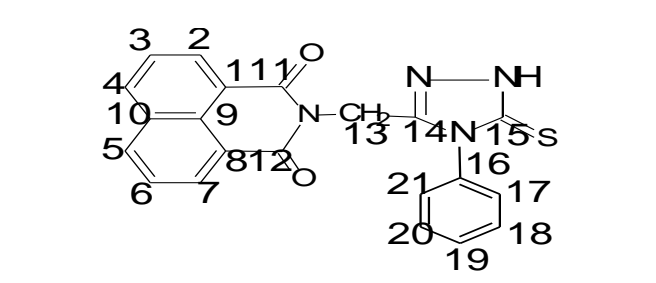 & $\begin{array}{c}48.41\left(\mathrm{C}_{13}\right) \\
\left.\text { 116.03-131.27( } \mathrm{C}_{1-}-\mathrm{C}_{10}\right) \text { and }\left(\mathrm{C}_{16}-\mathrm{C}_{21}\right) \\
157.47\left(\mathrm{C}_{14}\right), 160.29\left(\mathrm{C}_{11}, \mathrm{C}_{12}\right) \\
169.83\left(\mathrm{C}_{15}\right)\end{array}$ \\
\hline
\end{tabular}




\section{Antimicrobial Screening}

Selected of some newly synthesized naphthalimides linked to five membered heterocyclic rings were screened in vitro for their antibacterial activity against four types of pathogenic bacterial isolates and for antifungal activity against one type of monilia. DMSO as a blank exhibited no antimicrobial activity against any of the tested microorganisms used. The bacterial isolates were more susceptible to the synthesized compounds than isolated fungal. The recorded inhibition zones are summarized in Table (5).

Table (5)

Antimicrobial activity of selected compounds.

\begin{tabular}{|c|c|c|c|c|c|c|c|c|c|c|c|c|c|c|c|}
\hline \multirow[b]{2}{*}{$\begin{array}{l}\text { Comp. } \\
\text { No. }\end{array}$} & \multicolumn{3}{|c|}{$\begin{array}{c}\text { Staphylococcus } \\
\text { aureus } \\
\text { Concentrations } \\
(\text { mg/ml }) \\
\text { Inhibition zone } \\
\text { diameter }(\mathrm{mm})\end{array}$} & \multicolumn{3}{|c|}{$\begin{array}{l}\text { Bacillus subtilis } \\
\text { Concentrations } \\
\quad(\mathrm{mg} / \mathrm{ml}) \\
\text { Inhibition zone } \\
\text { diameter }(\mathrm{mm})\end{array}$} & \multicolumn{3}{|c|}{$\begin{array}{c}\text { E. Coli } \\
\text { Concentrations } \\
(\mathrm{mg} / \mathrm{ml}) \\
\text { Inhibition zone } \\
\text { diameter }(\mathrm{mm})\end{array}$} & \multicolumn{3}{|c|}{$\begin{array}{c}\text { Pseudomonas } \\
\text { aeuroginosa. } \\
\text { Concentrations }(\mathrm{mg} / \mathrm{ml}) \\
\text { Inhibition zone diameter } \\
(\mathrm{mm})\end{array}$} & \multicolumn{3}{|c|}{$\begin{array}{l}\text { Candida Albicans } \\
\text { Concentrations } \\
(m g / m l) \\
\text { Inhibition zone } \\
\text { diameter }(\mathbf{m m})\end{array}$} \\
\hline & 100 & 50 & 25 & 100 & 50 & 25 & 100 & 50 & 25 & 100 & 50 & 25 & 100 & 50 & 25 \\
\hline 3 & 20 & 13 & 9 & 18 & 15 & 11 & 25 & 20 & 19 & 14 & 12 & 7 & 10 & 7 & - \\
\hline 4 & 19 & 15 & 10 & 23 & 19 & 16 & 21 & 18 & 11 & 12 & 9 & 8 & 12 & - & - \\
\hline 5 & 20 & 14 & 8 & 18 & 12 & 8 & 21 & 19 & 14 & - & - & - & 14 & 7 & - \\
\hline 7 & 22 & 18 & 14 & 18 & 14 & 12 & 26 & 20 & 13 & 17 & 15 & 13 & 15 & 12 & 10 \\
\hline 8 & 20 & 16 & 13 & 20 & 16 & 10 & 23 & 17 & 15 & 17 & 16 & 12 & 17 & 12 & 7 \\
\hline 9 & 24 & 20 & 17 & 22 & 21 & 15 & 22 & 18 & 15 & 13 & 12 & 10 & 17 & 14 & 11 \\
\hline 11 & 18 & 9 & 7 & 13 & 10 & 8 & 14 & 12 & 8 & 15 & - & - & 19 & 16 & 14 \\
\hline 13 & 25 & 21 & 17 & 21 & 20 & 18 & 22 & 15 & 12 & 13 & - & - & 20 & 17 & 15 \\
\hline 15 & 20 & 16 & 8 & 22 & 16 & 14 & 23 & 19 & 18 & 12 & - & - & 22 & 17 & 14 \\
\hline 17 & 22 & 20 & 18 & 20 & 17 & 15 & 19 & 16 & 10 & 15 & 7 & - & 21 & 18 & 17 \\
\hline $\begin{array}{c}\text { Sulfamethoxazole } \\
\text { (std.) }\end{array}$ & 32 & 28 & 22 & 34 & 26 & 20 & 31 & 24 & 21 & 29 & 20 & 18 & $*$ & $*$ & $*$ \\
\hline $\begin{array}{l}\text { Clotrimazole } \\
\text { (std.) }\end{array}$ & $*$ & $*$ & $*$ & $*$ & $*$ & $*$ & $*$ & $*$ & $*$ & $*$ & $*$ & $*$ & 26 & 24 & 22 \\
\hline
\end{tabular}

$*$ not tested.

\section{- = no inhibition zone.}

We observed some important results from the data of inhibition zone:

Most of the synthesized compounds showed antibacterial and/or antifungal activities. All compounds at concentration (100 mg/ml) were highly active against Staphylococcus aureus except $(4,11)$ showed moderate activity.

Most compounds at concentration $(100 \mathrm{mg} / \mathrm{ml})$ were highly active against
Bacillus subtilis whereas $(3,5,7,11)$ showed moderate activity against this microorganism.

All compounds at concentration $(100 \mathrm{mg} / \mathrm{ml})$ except $(11,17)$ showed highly active against E. Coli. Gram (-ve) type Pseudomonas aeuroginosa showed resistance to compound (5) at all concentrations and to compounds $(11,13,15,17)$ at lower concentrations. Other compounds showed moderate to low activity against this bacterial 
isolate. Compounds $(11,13,15,17)$ acts as good antifungal agents towards Candida Albicans. While other Compounds show moderate low activity especially at concentrations 50 and $25(\mathrm{mg} / \mathrm{ml})$. Therefore triazole compounds $(11,13,15,17)$ can be recommended for further studies.

\section{References}

[1] Cechinel-Filho V., Campos F., Correa R., Nunes J., Yunes R., "Chemical aspects and therapeutic potential of cyclic imides", Quim. Nova., 26,230-241, 2003.

[2] Lv M., Xu H., "Overview of naphthalimide analogs as anticancer agents", Curr. Med. Chem., 16, 4797-4813, 2009.

[3] Fuente R., Sonawane N., Arumainayagam D., Verkman A.S., "Small molecules with antimicrobial activity against E. coli and P. aeruginosa identified by high-throughput screening", Br. J. Pharmacol., 149, 551559, 2006.

[4] Muth M., Hoerr V., Glaser M., Ponte A., Moll H., Stich A., Holzgrabe U., "Antitrypanosomal activity of quaternary naphthalimide derivatives", Bioorg. Med. Chem.lett., 17, 1590-1593, 2007.

[5] Andricopulo A.D., Muller A.L., Filho V.C., Cani G.S., Roos J.F., Correa R., Santos A., Yunes R., "Analgesic activity of cyclic imides: 1,8-naphthalimide and 1,4,5,8-naphthalenediimide derivatives", IL Farmaco, 55,319-321, 2000.

[6] Bailly C., Carrasco C., Joubert A., Bal C., Wattez N., Hildebrand M., Lansiaux A., Colson P., Houssier C., Cacho M., Ramos A., Brana M., "Chromophore-modified bisnaphthalimides:DNA recognition, topoisomerase inhibition, and cytotoxic properties of two monobisfuronaphthalimides", Biochemistry, 42, 4136-4150, 2003.

[7] Sule E., Serdar O., Esin E., "Synthesis and photophysical characterizations of thermal stable naphthalene benzimidazoles", $J$. Fluoresc., 21, 1565-1573, 2011.

[8] Sumitra C., Yogesh B., Shipra B., "Synthesis and antibacterial activity of some new triazole derivatives", Arch. Apll. Sci. Res., 2 (3), 117-126, 2010.

[9] Christian S., Dougal J., Amani A., John E., "Silver mediated one-step synthesis of oxazoles from $\alpha$-haloketones", J.S.Chem.Soc., 15, 375-378, 2011.

[10] Prokopenko V., Pilo S., Brovarets V., "Synthesis of 4-hetaryl-substituted 5amino and 5-sulfanyl-1,3-oxazole derivatives", RUSS. J. G. CHEM., 81(2), 307-312, 2011.

[11] Bhaskar S., and Shankaraiah G., "A versatile multicomponent one-pot synthesis of thiazole derivatives under solvent free conditions: designed by pass showed antiviral activity as predicted", Int. J. Pharm.Sci.Rev. \& Res., 3(2), 96-98, 2010.

[12] Raghav M., Isha T., Priyanka N., Sharma K., "Synthesis and antimicrobial evaluation of some novel thiazole derivatives", Der. Pharmacia Sinica, 3(3), 361-366, 2012.

[13] Wagnat W., Mohamed A., Faten I., Salama A., "New approaches for the synthesis of thiazoles and their fused derivatives with antimicrobial activities", J. Chin. Chem. Soc., 55, 1133-1144, 2008.

[14] Olgad C., Stefaniaf B., Gabriel S., Constantin D., "Synthesis and characterization of some 1,2,4-triazole-3thiones obtained from intramolecular cyclization of new 1-(4-(4-Xphenylsulfonyl)benzoyl)-4-(4-iodophenyl)3-thiosemicarbazides", J.Serb. Chem. Soc., 75 (11), 1463-1471, 2010.

[15] Paulvannan K., Tao C., Ron H., "An improved synthesis of 1,2,4-triazoles using $\mathrm{Ag}_{2} \mathrm{CO}_{3} "$, Tetrahedron, 56, 8071-8076, 2000 .

[16] Kamaladin G., Mokhtar A., Shohre R., Hajir B., Barahman M., Niyaz M., "Synthesis and characterization of novel monoazo N-ester-1,8-naphthalimide disperse dyestuffs", J. Chin. Chem. Soc., 54(4), 1021-1028, 2007.

[17] Wafaa W., "Synthesis of some new derivatives of 1,2,4-triazole and thiazole from 1,2,3,4-tetrahydrocarbazole and study their biological activity", J. Al-Nahrain Univ.Sci.,13(2), 54-62, 2010.

[18] Suaad M. Al-Majidi and Zainab A. AlMessri, "Synthesis of some new substituted 1,2,4-triazole and 1,3,4thiadiazole and study their activities on 
some strains of bacteria", J.Al-Nahrain Univ.Sci., 10 (1), 30-37, 2007.

[19] Zainab A. Al-Messri, "Synthesis of some1,2,4-triazoles derived from 2mercapto benzimidazole", J.Al-Nahrain Univ.Sci., 6(1), 200-208, 2009.

[20] Anesini C., Perez C., "Screening of plants used in argentine folk medicine for antibacterial activity", $J$. Ethnropharmacol., 3, 35-47, 1993.

[21] Bojinov V., Ivanova G., Chovelon J., Grabchev I., "Photophysical and photochemical properties of some 3bromo-4-alkylamino-N-alkyl-1,8-

naphthalimides", Dyes \& Pigments., 58, 65-71, 2003.

[22] Middleton R., Parrick J., "Preparation of 1,8-naphthalimides as candidate fluorescent probes of hypoxic cells", $J$. Het. Chem., 22, 1567-1572, 1985.

[23] Ralph,L. Christine,K. Hermann,T. Morrill,D. Curtin,R. "The Systematic Identification of Organic Compounds" $8^{\text {th }}$ ed. John wiley\&sons. Inc., 253-256, 2004.

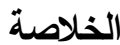

تضمن البحث تحضير مشتقات حلقية غير متجانسة

جديدة لـ 8,1- نفتالئميدات التي تحمل معوضات اوكسازول، ثايازول او ترايازول كما يلي :

التفاعل المباشر لـ 8,1- حامض النفتالك اللامائي مع لرع

كلايسينات الاثيل في ثنائي مثيل السلفوكسيد كمذيب تحت التصعيد وعند درجة حرارة عالية لمدة ستة عشر ساعة لبنتج

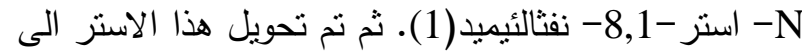
مشتقات (اليوريا، الثايويوريا، السيميكاربازايد، فينيل سيميكاربازايد، الثايوسيميكاربازايد وفينيل ثايوسيميكاربازايد) وذللك من خلال التفاعل مع (اليوريا، الثايويوريا، السيميكاربازايد، فينيل سيميكاربازايد، الثايوسيميكاربازايد وفينيل ثايوسيميكاربازايد) على التوالي وتم الحصول على المركبات

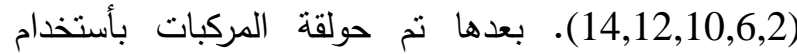
مختلف الكواثف. الحولقة الاولى للمركبات (6,2) بأستخدام بروميدالفيناسيل المعوض في الموقع بارا اعطى مشتقات الاوكسازول (3-5) ومشتقات الثايازول (7-9) على التوالي. علاوة على ذلك مشتقات الترايازول حضرت من خلال الغلق الحلقي الثاني للمركبات (16,14,12,10) في وسط قاعدي
من هيدروكيد الصوديوم بتركيز (4N) ليعطي المركبات (17,15,13,11) على التوالي. تراكيب المركبات المحضرة الجديدة شخصت من خلا الطرق الطيفية H-,FTIR م ${ }^{13}$ وبعض الخواص الفيزيائية واجراء N-NMR بعض الكثوفات النوعية حيث كانت النتائج المستحصلة مطابقة للتراكيب المقترحة. المركبات المحضرة اختبرت فعاليتها المضادة للميكروبات بثلاث تراكيز مختلفة خارج جسم الكائن الحي ضد نوعين البكتريا المرضية موجبة الصبغة ونوعين اخرين سالبة الصبغة ونوع من الفطريات وقد اظهرت النتائج فعالية جيدة الى منوسطة ضد انواع الاحياء

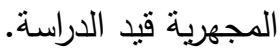

